\title{
DLC-1 is an independent prognostic marker and potential therapeutic target in hepatocellular cancer
}

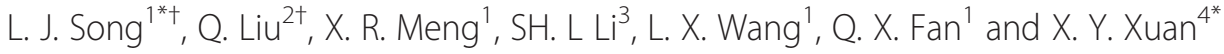

\begin{abstract}
Background: The 5-year survival rate of patients with hepatocellular cancer (HCC) was very low because of invasion and metastasis in the early stage. Biomarkers might help predict early occurrence of invasion and metastasis. Accumulating evidence has shown that deleted in liver cancer-1 (DLC1) may be considered as a metastasis suppressor gene in numerous solid and hematological cancers. However, its prognostic role and mechanisms that regulate and coordinate these activities remain poorly understood.

Methods: With the method of immunohistochemistry, the expression of DLC-1 as well as Rho A, ROCK2, moesin had been characterized in $80 \mathrm{HCC}$ tissues and adjacent noncancerous tissues. The correlation between their expression and their relationships with clinicopathological characteristics of HCC were also investigated. In addition, the prognostic value of DLC1 expression within the tumor tissues was assessed by Cox regression and Kaplan-Meier analysis.

Results : DLC1 expression was significantly lower in HCC tissues than in adjacent noncancerous tissues, and DLC-1 expression was found to be negatively correlated with tumor differentiation, TNM stage and lymph node metastasis. Furthermore, DLC-1 expression was found to inversely correlate with Rho A, ROCK2 and moesin which were all highly expressed in HCC tissues. Kaplan-Meier analysis showed that significantly longer 5-year survival rate was seen in HCC patients with higher DLC1 expression, compared to those with lower expression of DLC1. Multivariate Cox proportional hazard analyses revealed that DLC1 was an independent factor affecting the overall survival probability.

Conclusion: DLC1 could be served as a tumor suppressor gene in the progression especially in the invasion and metastasis of HCC. DLC1 perhaps played its role by regulating the expression of Rho A, ROCK2 and moesin. Evaluation of the expression of DLC-1 might be a good prognostic marker for patients with HCC.
\end{abstract}

Keywords: HCC, DLC-1, Rho A, ROCK2, moesin

\section{Background}

Liver cancer is one of the most common malignancies in China and the 5-year survival rate of it is very low because of invasion and metastasis in the early stage [1,2]. Mechanisms of invasion and metastasis are diverse and not yet clear-cut in most cases. So the identification of indicators or markers that may help us to assess tumor behavior of invasion and metastasis are very important for us [3].

\footnotetext{
* Correspondence: lijiesongcn@hotmail.com; xxy@zzu.edu.cn

${ }^{\dagger}$ Equal contributors

'Department of Oncology, the first affiliated hospital of Zhengzhou University, Henan 450000, China

${ }^{4}$ Department of Microbiology and Immunology, Basic Medical School of Zhengzhou University, 100 Kexue Road, Zhengzhou, Henan 450001, China Full list of author information is available at the end of the article
}

Deleted in liver cancer-1 (DLC-1) was first cloned by using subtractive hybridization method in human hepatocellular carcinomas and later it was identified as a breast cancer metastasis suppressor in microarray comparisons between breast cancer cell lines $[4,5]$. Then, deletion or promoter methylation of DLC-1 has been described in multiple cancers. DLC-1 is a RhoGAP (GTPase-activating protein) that inhibits/inactivates Rho-dependent signal transduction $[4,6]$. While Rho is key factors in cell proliferation, polarity, cytoskeletal remodeling and migration, the aberrant function of their regulators may lead to cell transformation $[6,7]$. Data showed that DLC-1 mRNA expression was lost in $95 \%$ of patient with NSCLC tumors tissues and $58 \%$ of NSCLC cell lines, due at least in part through its function as 
a RhoGAP and thus negatively regulating the expression of RhoA and related RhoB, RhoC [8]. Nevertheless, a detection of DLC-1 as a therapeutic indicator or prognostic markers in liver cancers have litter been elucidated and the role and mechanism of DLC-1 in liver cancer especially in the invasion and metastasis process remains to be fully eclucidated.

There is considerable and growing evidence for the importance of the ROCK gene in actomyosin contractility, focal adhesion assembly, cytokinesis and cell proliferation $[9,10]$. ROCK has also been implicated in colorectal [11], breast [12], gastric [13], and hepatocellular cancer metastatic growth [14]. One identified mechanism for ROCK activation in cancer involves the loss of function of the DLC-1, which encodes a GTPase activating protein (RhoGAP) for the RhoA and RhoC small GTPases [15].

Moesin, a member of the ERM (moesin, radixin, ezrin) family of proteins has been reported to be overexpressed in many kinds of cancers $[16,17]$. Leroi et al. [18] sowed that moesin not ezrin and radixin was up-regulated in glioblastoma multiforme in comparison to non-malignant brain tissue samples. In addition, $\mathrm{He}$ [19] et al. reported that the extracellular small GTPase RhoA/ROCK-2 cascade mediated the increased moesin expression and phosphorylation, however, the relationship between DLC-1 and moesin in liver cancer has not been evaluated before.

In the current study, the relationship between the expression of DLC1 and various clinicopathologic parameters and immunohistochemical markers, such as RhoA, ROCK2 and Moesin were analyzed. Furthermore, the prognostic significance of DLC1 in human HCC was also explored.

\section{Methods}

\section{Patient specimens}

This study was approved by the Ethics Committee of the First Affiliated Hospital of Zhengzhou University (Zhengzhou, Henan, China). Written consents were obtained from all those patients included in this study. A total of 80 formalin-fixed paraffin-embedded liver cancer tissues and the corresponding normal tissues were collected from the archive of the First Affiliated Hospital of Zhengzhou University from 2009 to 2013. None of these patients received radiotherapy or chemotherapy prior to surgery. Briefly, the 80 patients consisted of 19 females and 61 males with ages ranging between 36 and 78 years old (mean age: 65 years old). According to the newer 2010 TNM classification of malignant tumours, liver cancers were staged into 54 I-II and 26 III stages. The histological grade of tumor differentiation was assigned into 61 I-II stage and 29 III-IV stage. Cancer size was calculated by measuring the largest dimension of the cancer specimen. 34 cases localized liver cancers without dissemination in liver and portal vein invasion were viewed as low invasion groups while other 46 cases with dissemination in liver and portal vein invasion were viewed as high.

\section{Immunohistochemistry}

The immunohistochemistry protocols were described previously [20]. Primary antibodies against DLC-1, Rho A, ROCK2 and Moesin were used to detect the corresponding proteins. Briefly, slides were incubated with $1.5 \% \mathrm{H}_{2} \mathrm{O}_{2}$ at room temperature for 20 min to eliminate the endogenous peroxidase activity. Then the slides were incubated with a solution comprising $0.2 \%$ triton-X 100 and $5 \%$ goat serum at room temperature for $2 \mathrm{~h}$ to quench immunoglobulin's non-specific binding. After that, the primary antibodies were added to the slides individually and incubated overnight at $4{ }^{\circ} \mathrm{C}$. Next day, secondary antibody was applied to the slides and incubated at room temperature for $2 \mathrm{~h}$. At last, the immune complexs were visualized by incubating the sections with DAB. The slides were counterstained with hematoxylin and mounted. Negative controls were identically treated with the primary antibodies by PBS.

\section{Immunohistochemistry evaluation}

Immunostained sections were evaluated independently by two pathologists. Immunostaining was evaluated semiquantitatively by estimating immunoreactivity extent and intensity. Staining extent was rated according to the percentage of positive cells. Samples with no stained tumor cells were rated as 0 , those with $<10 \%$ of stained tumor cells were rated as 1 , those $11-50 \%$ of stained tumor cells were rated as 2 , those with $50-75 \%$ of stained tumor cells were rated as 3 and those with $>75 \%$ of stained tumor cells were rated as 4 . Staining intensity was graded as follows: negative (0), weak (1), moderate (2) and strong (3). The overall scores were the product of staining intensity multiplied the staining extent. An overall staining score of $0-3$ and $>3$ were regarded as negative $(-)$ and positive $(+)$ protein expression, respectively.

\section{Statistical analyses}

The significant difference of DLC-1, Rho A, ROCK2 and moesin gene expression between specimen groups was carried out using the unpaired Student's $t$-test. The survival rates after tumor removal were calculated by the Kaplan-Meier method, and differences in survival curves were analyzed by the Log-rank tests. Multivariate survival analysis was performed on all the significant characteristics measured by univariate survival analysis through the Cox proportional hazard regression model. Differences were considered statistically significant when the $P$-value was $<0.05$.

\section{Results}

The expression of DLC-1, Rho A, ROCK2 and moesin proteins in liver cancer tissues

The cellular locations of DLC-1, Rho A, ROCK2 and moesin proteins were all mainly found in the cytoplasm. 

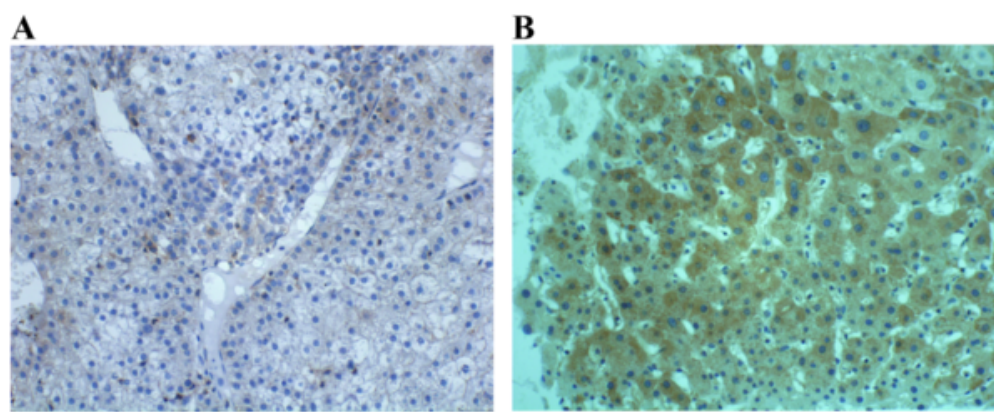

Fig. 1 The expression of DLC-1 in HCC tumor and adjacent tissues. a Cytoplasm staining of DLC-1 in HCC tumor tissues ( $\times 400)$. b Cytoplasm staining of DLC-1 in adjacent normal tissues $(\times 400)$

As shown in Figs. 1a, b; 2a, b; 3a, b and 4a, b, positive staining rates of DLC-1, Rho A, ROCK2 and moesin in liver cancer tissue samples were $38.75,78.75,75.00$ and $86.25 \%$ respectively, which was significantly different from those in normal mucosa $(83.75,43.75,40.00$ and $66.25 \%)(P<0.001$, Table 1$)$.

\section{Clinicopathological significance of the expression of DLC-1,} Rho A, ROCK2 and moesin proteins in liver cancer tissues Clinicopathological significance of the expression of DLC1, Rho A, ROCK2 and moesin proteins in liver cancer tissues are shown in Table 2. We found a negative correlation between DLC-1 expression and Tumor differentiation $(p<0.001)$ and TNM stage $(p<0.001)$.In addition, the expression of DLC-1 in low invasion groups was higher than those in high invasion groups $(p<0.001)$.

While the expression levels of Rho A, ROCK2 and moesin were positively correlated with Tumor differentiation, and TNM stage. Furthermore, Rho A, ROCK2 and moesin expression levels were significantly higher in $\mathrm{HCC}$ cancer patients with high invasion ability.

\section{Association between DLC-1 and Rho A, ROCK2, moesin expression in HCC cancer tissues}

Spearman's rank correlational analysis revealed a significant negative correlation between the expression levels of DLC1 and Rho A, ROCK2, moesin ( $r=-0.963,-0.669,-0.631)$.

\section{Survival analysis}

HCC patients with low DLC1 expression had lower disease-free and 5-year survival rates than those with high DLC1 expression as determined using the Kaplan-Meier method (Fig. 5). Univariate Cox regression analysis also identified that clinical variables including gender, tumor number, maximal tumor size, HBV infection, tumor differentiation, TNM stage and portal vein invasion and dissemination were all positively correlated with overall survival. Furthermore, to evaluate the potential of DLC1 expression as an independent prognostic marker for overall survival of HCC, multivariate Cox regression analyses were performed. While the others failed to demonstrate independence, DLC1 expression (HR: 2.234, 95 \% CI: $0.937-4.148, \quad p<0.001$ ), tumor differentiation (HR: $3.769,95 \% \mathrm{CI}: 1.925-6.989, \mathrm{P}=0.003)$, and portal vein invasion and dissemination (HR: 1.341,95 \% CI: $0.843-$ 2.631, $p<0.001)$ may play a role in predicting the overall survival in $\mathrm{HCC}(p<0.05)$.

\section{Discussion}

$\mathrm{HCC}$ is one of the most common malignancies worldwide. Despite advances in the detection and treatment of HCC, the mortality rate remains high because of early occurrence of invasion and metastasis [21]. Hence to improve early detection of invasion and metastasis and their molecular characterization in $\mathrm{HCC}$, to develop interventions
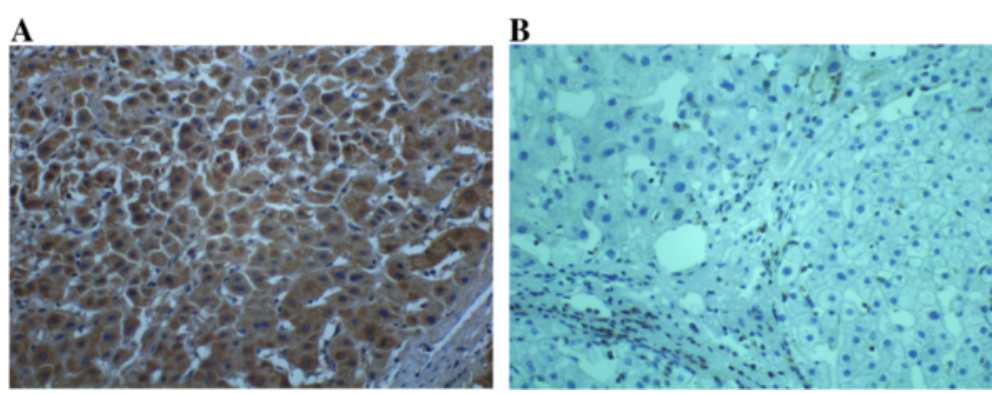

Fig. 2 The expression of RhoA in HCC tumor and adjacent tissues. a Cytoplasm staining of RhoA in HCC tumor tissues (×400). b Cytoplasm staining of RhoA in adjacent normal tissues $(\times 400)$ 

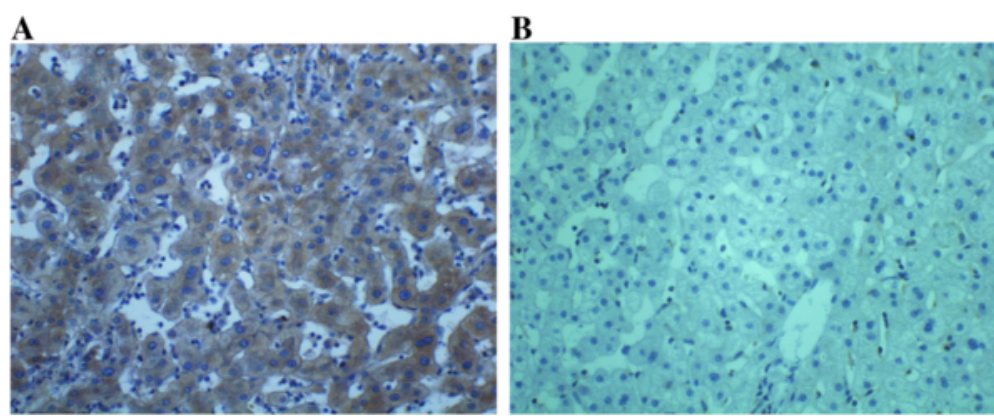

Fig. 3 The expression of ROCK2 in HCC tumor and adjacent tissues. a ytoplasm staining of ROCK2 in HCC tumor tissues ( $\times 400)$. b Cytoplasm staining of ROCK2 in adjacent normal tissues $(\times 400)$

that are targeted at blocking or reversing the invasion and metastasis process are very important for us.

DLC-1 has been known as tumor suppressor gene in many kinds of cancers including liver, lung, breast, brain, prostate and colon cancers [22, 23]. And re-expression of DLC- 1 in cancer cells regulates the structure of actin cytoskeleton and focal adhesions and significantly inhibits cell growth, colony formation and invasion capacity, supporting its role as a tumor suppressor [24]. Thus, DLC-1 has the potential to be a key therapeutic indicator or markers for cancer gene therapy. In the present study, DLC-1 was found to be lower expressed in HCC tissues. DLC-1 positive expression was observed in 31 out of $80 \mathrm{HCC}$ patients. And the lower expression of DLC-1 was correlated negatively with tumor differentiation and TNM stage. More importantly, the lower expression of DLC-1 found to be negatively linked with aggressive clinical behavior. HCC tissues with dissemination in liver and portal vein invasion showed lower DLC-1 expression than those in localized tissues without dissemination in liver and portal vein invasion.

Regarding DLC1 expression, recent reports have shown a growing interest in its prognostic impact in terms of survival. In this study, the prognosis of HCC patients with a low expression of DLC1 was poor, and Cox regression analysis indicated that low expression level of DLC1 was a significant prognostic factor for a poor survival rate of HCC patients. These findings raised the possibility that lower expression of DLC1 could be a factor of worst disease-specific survival, independently of any other variables.

DLC-1 was also named ARHGAP7 because it contains a RhoGTPase activating protein (RhoGAP) domain and these domains can convert the active GTP-bound Rho proteins to the inactive GDP-bound state and negatively regulate Rho GTPases [25]. RhoA is one of the bestknown members of RhoGAP. ROCKs were originally isolated as downstream targets of Rho A [26]. RhoA binding with the C-terminal domain of ROCK, forming the Rho A/ROCK pathway, and controls a wide variety of cellular processes dependent on the re-arrangement of the actin cytoskeleton and changes of cell contractility. Yet, ROCK does not directly act on cytoskeletal molecules and ERM (radixin/ezrin/moesin) proteins are emerged as the candidates that likely linking the activation of ROCK and the cytoskeleton reorganization [27, 28]. Moesin is one of the most important and it has been reported that Rho A/ROCK is a typical upstream pathway for the phosphorylation of moesin [29, 30]. In the present study, Rho A and ROCK2 expression were found to negatively correlated with DLC-1 expression. Rho A and ROCK2 expression were identified in 63 and 60 of
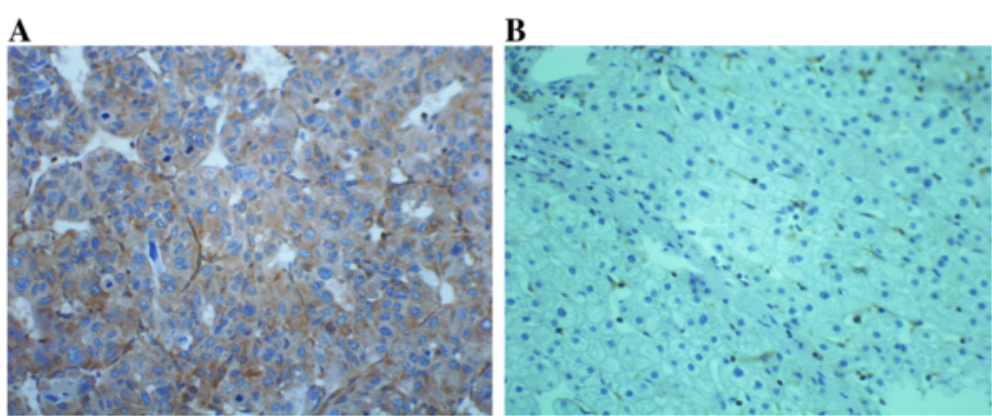

Fig. 4 The expression of moesin in HCC tumor and adjacent tissues. a Cytoplasm staining of moesin in HCC tumor tissues ( $\times 400)$. b Cytoplasm staining of moesin in adjacent normal tissues $(\times 400)$ 
Table 1 The expression of DLC-1, RhoA, ROCK2 and moesin in HCC tumor and adjacent tissues

\begin{tabular}{lllll}
\hline & & $\begin{array}{l}\text { HCC tissues } \\
(n=80)\end{array}$ & $\begin{array}{l}\text { Adjacent liver tissues } \\
(n=80)\end{array}$ & $p$ \\
\hline DLC-1 & High & 31 & 67 & $<0.001$ \\
& Low & 49 & 13 & $<0.001$ \\
RhoA & High & 63 & 35 & $<0.001$ \\
& Low & 17 & 45 & \\
\multirow{2}{*}{ ROCK2 } & High & 60 & 32 & $<0.001$ \\
& Low & 20 & 48 & \\
moesin & High & 69 & 53 & \\
& Low & 11 & 27 & \\
\hline
\end{tabular}

the HCC cases. Expression of Rho A and ROCK2 were all correlated positively tumor differentiation and TNM stage dissemination in liver and portal vein invasion. Furthermore, positive expression of Rho A and ROCK2 were also linked with dissemination in liver and portal vein invasion of HCC tissues. These data indicated that DLC-1, Rho A and ROCK2 perhaps together played an important role in the invasion and metastasis of $\mathrm{HCC}$, and that DLC-1 perhaps played as a tumor suppressor by inhibiting the activation of Rho A/ROCK2 pathway . Furthermore, we found that the expression of phosphorylation of moesin was negatively correlated with the expression of DLC-1. Positive expression of phosphorylation of moesin was all correlated positively with tumor differentiation, TNM stage and dissemination in liver and portal vein invasion. So DLC-1 perhaps exerted its effects in invasion in $\mathrm{HCC}$ by regulating the expression of moesin through the RhoA/ROCK pathway.

Regarding DLC1 expression, recent reports have shown a growing interest in its prognostic impact in terms of survival. In this study, the prognosis of HCC patients with a low expression of DLC1 was poor, and Cox regression analysis indicated that low expression level of DLC1 was a significant prognostic factor for a poor survival rate of $\mathrm{HCC}$ patients. These findings raised the possibility that lower expression of DLC1 could be a factor of worst disease-specific survival, independently of any other variables.

Table 2 The relationship between DLC-1, RhoA, ROCK2, moesin and clinicopathological variables in HCC tissues

\begin{tabular}{|c|c|c|c|c|c|c|c|c|c|c|c|c|}
\hline \multirow[t]{2}{*}{ Clinicopatholog-ical variables } & \multicolumn{3}{|c|}{ DLC1 expression } & \multicolumn{3}{|c|}{ RhoA expression } & \multicolumn{3}{|c|}{ Rock2 expression } & \multicolumn{3}{|c|}{ moesin expression } \\
\hline & $\mathrm{H}$ & $L$ & $p$ & $\mathrm{H}$ & $\mathrm{L}$ & $p$ & $\mathrm{H}$ & $L$ & $\mathrm{p}$ & $\mathrm{H}$ & $L$ & $\mathrm{p}$ \\
\hline \multicolumn{13}{|l|}{ Gender } \\
\hline Male & 23 & 38 & 0.731 & 48 & 13 & 0.98 & 46 & 15 & 0.879 & 55 & 8 & 0.29 \\
\hline Female & 8 & 11 & & 15 & 4 & & 14 & 5 & & 15 & 4 & \\
\hline \multicolumn{13}{|l|}{ Age (years) } \\
\hline$<45$ & 9 & 14 & 0.958 & 17 & 6 & 0.502 & 17 & 6 & 0.887 & 19 & 4 & 0.548 \\
\hline$\geq 45$ & 22 & 35 & & 46 & 11 & & 43 & 14 & & 50 & 7 & \\
\hline \multicolumn{13}{|l|}{ Tumor number } \\
\hline Singel & 20 & 30 & 0.767 & 40 & 10 & 0.724 & 39 & 11 & 0.424 & 43 & 7 & 0.933 \\
\hline Multiple & 11 & 19 & & 23 & 7 & & 21 & 9 & & 26 & 4 & \\
\hline \multicolumn{13}{|l|}{ Maximal tumor size $(\mathrm{cm})$} \\
\hline$<5$ & 19 & 30 & 0.995 & 38 & 11 & 0.742 & 37 & 13 & 0.789 & 43 & 6 & 0.623 \\
\hline$\geq 5$ & 12 & 19 & & 25 & 6 & & 23 & 7 & & 26 & 5 & \\
\hline \multicolumn{13}{|l|}{ Tumor differentiation } \\
\hline$|-| \mid$ & 12 & 49 & $<0.001$ & 36 & 15 & 0.018 & 33 & 18 & $<0.001$ & 41 & 10 & 0.043 \\
\hline III-IV & 19 & 10 & & 27 & 2 & & 27 & 2 & & 28 & 1 & \\
\hline \multicolumn{13}{|l|}{ HBV infection } \\
\hline No & 7 & 11 & 0.989 & 14 & 4 & 0.909 & 13 & 5 & $<0.001$ & 15 & 3 & 0.683 \\
\hline Yes & 24 & 38 & & 49 & 13 & & 47 & 15 & & 54 & 8 & \\
\hline \multicolumn{13}{|l|}{ TNM stage } \\
\hline$|-| \mid$ & 11 & 43 & $<0.001$ & 39 & 15 & 0.034 & 37 & 17 & 0.002 & 44 & 10 & 0.074 \\
\hline III & 20 & 6 & & 24 & 2 & & 23 & 3 & & 25 & 1 & \\
\hline \multicolumn{13}{|c|}{ Portal vein invasion and dissemination } \\
\hline Yes & 8 & 38 & $<0.001$ & 39 & 7 & $<0.001$ & 40 & 6 & 0.004 & 43 & 3 & 0.029 \\
\hline No & 23 & 11 & & 14 & 20 & & 20 & 14 & & & & \\
\hline
\end{tabular}




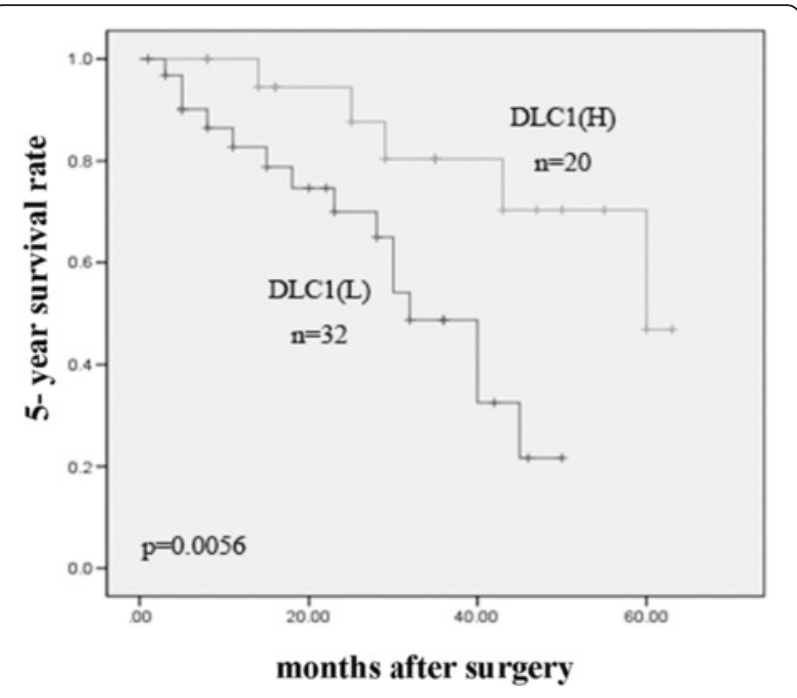

Fig. 5 Kaplan-Meier analyses of 5-year survival rates in 52 HCC patients in relation to DLC-1 protein overexpression. Patients with HCC with low DLC-1 expression had lower 5 -year $p<0.001$ ) survival rates than those with high DLC-1 expression as determined using the Kaplan-Meier method.(H: high DLC-1 expression, L: low DLC-1 expression)

\section{Conclusion}

DLC-1 appeared to also play as a tumor suppressor gene in HCC. And DLC-1 might work its way by negatively regulating the expression of RhoA/ROCK2/ moesin. DLC-1 itself could be a direct indicator or marker for the invasion and prognosis of HCC.

\section{Competing interests}

The authors declare that there are no conflicts of interest.

\section{Authors' contributions}

$\sqcup \mathrm{S}$ and $\mathrm{QL}$ are responsible for laboratory work, data analysis and paper writing. XR M is responsible for laboratory work and data analysis. SL L is responsible for sample collection and clinical data analyses. LX W and QX F are responsible for project design. $\mathrm{XY} X$ is responsible for project design and manuscript preparation. All authors read and approved the final manuscript.

\section{Acknowledgements}

This work was supported by grants from the Technique Foundation of Henan (152102310264).

\section{Author details}

'Department of Oncology, the first affiliated hospital of Zhengzhou University, Henan 450000, China. ${ }^{2}$ Department of Neurosurgery, the fifth affiliated hospital of Zhengzhou University, Henan 450000, China. ${ }^{3}$ Department of Pathology, the first affiliated hospital of Zhengzhou University, Henan 450000, China. ${ }^{4}$ Department of Microbiology and Immunology, Basic Medical School of Zhengzhou University, 100 Kexue Road, Zhengzhou, Henan 450001, China.

\section{Received: 26 October 2015 Accepted: 28 January 2016}

Published online: 04 February 2016

\section{References}

1. Cai ZQ, Si SB, Chen C, Zhao Y, Ma YY, Wang L, et al. Analysis of prognostic factors for survival after hepatectomy for hepatocellular carcinoma based on a bayesian network. PLoS One. 2015;10(3):e0120805.
2. Wang W, Li GY, Zhu JY, Huang DB, Zhou HC, Zhong W, et al. Overexpression of AGGF1 is correlated with angiogenesis and poor prognosis of hepatocellular carcinoma. Med Oncol. 2015;32(4):131.

3. Będkowska GE, Ławicki S, Gacuta E, Pawlowski P, Szmitkowski M. M-CSF in a new biomarker panel with HE4 and CA 125 in the diagnostics of epithelial ovarian cancerpatients. J Ovarian Res. 2015;8(1):27.

4. Holeiter G, Heering J, Erlmann P, Schmid S, Jähne R, Olayioye MA. Deleted in liver cancer 1 controls cell migration through a Dia1-dependent signaling pathway. Cancer Res. 2008;68(21):8743-51.

5. Ullmannova V, Popescu NC. Inhibition of cell proliferation, induction of apoptosis, reactivation of $\mathrm{DLC1}$, and modulation of other gene expression by dietary flavone in breast cancer cell lines. Cancer Detect Prev. 2007;31(2):110-8.

6. Braun AC, Olayioye MA. Rho regulation: DLC proteins in space and time. Cell Signal. 2015;27(8):1643-51.

7. Ravi A, Kaushik S, Ravichandran A, Pan CQ, Low BC. Epidermal growth factor activates the Rho GTPase-activating protein (GAP) Deleted in Liver Cancer 1 via focal adhesion kinase and protein phosphatase 2A.J Biol Chem. 2015;290(7):4149-62.

8. Yuan BZ, Jefferson AM, Baldwin KT, Thorgeirsson SS, Popescu NC, Reynolds SH. DLC-1 operates as a tumor suppressor gene in human non-small cell lung carcinomas. Oncogene. 2004;23(7):1405-11.

9. Zhang J, He X, Ma Y, Liu Y, Shi H, Guo W, et al. Overexpression of ROCK1 and ROCK2 inhibits human laryngeal squamous cell carcinoma. Int J Clin Exp Pathol. 2015;8(1):244-51.

10. Julian L, Olson MF. Rho-associated coiled-coil containing kinases (ROCK): structure, regulation, and functions. Small GTPases. 2014;5(2):e29846.

11. Voorneveld PW, Kodach LL, Jacobs R, Liv N, Zonnevylle AC, Hoogenboom JP, et al. Loss of SMAD4 alters BMP signaling to promote colorectal cancer cell metastasis via activation of Rho and ROCK. Gastroenterol. 2014;147(1):196-208. e13.

12. Bhandary L, Whipple RA, Vitolo MI, Charpentier MS, Boggs AE, Chakrabarti KR Thompson KN, Martin SS. ROCK inhibition promotes microtentacles that enhance reattachment of breast cancer cells. Oncotarget. 2015;6(8):6251-66.

13. Matsuoka T, Yashiro M. Rho/ROCK signaling in motility and metastasis of gastric cancer. World J Gastroenterol. 2014;20(38):13756-66.

14. Lin L, Yang XM, Li J, Zhang YL, Qin W, Zhang ZG. Microfilament regulatory protein MENA increases activity of RhoA and promotes metastasis ofhepatocellular carcinoma. Exp Cell Res. 2014;327(1):113-22.

15. Wong CC, Wong CM, Ko FC, Chan LK, Ching YP, Yam JW, et al. Deleted in liver cancer 1 (DLC1) negatively regulates Rho/ROCK/MLC pathway in hepatocellularcarcinoma. PLoS One. 2008:3(7):e2779.

16. Deming PB, Campbell SL, Stone JB, Rivard RL, Mercier AL, Howe AK. Anchoring of protein kinase A by ERM (ezrin-radixin-moesin) proteins is required for proper netrin signaling through DCC (deleted in colorectal cancer). J Biol Chem. 2015; 290(9):5783-96.

17. Abiatari I, Esposito I, De Oliveira T, Felix K, Xin H, Penzel R et al. Moesindependent cytoskeleton remodelling is associated with an anaplastic phenotype of pancreatic cancer. J Cell Mol Med. 2010;14(5):1166-79.

18. DeSouza LV, Matta A, Karim Z, Mukherjee J, Wang XS, Krakovska O, et al. Role of moesin in hyaluronan induced cell migration in glioblastoma multiforme. Mol Cancer. 2013;12:74

19. Mian H, Yang C, Wen L, Liu Q, Liu J, Huang J, et al. Vascular endothelial growth factor $C$ promotes cervical cancer metastasis via up-regulation and activation of RhoA/ROCK-2/moesin cascade. BMC Cancer. 2010:10:170.

20. Smith SC, Baras AS, Owens CR, Dancik G, Theodorescu D. Transcriptional signatures of Ral GTPase are associated with aggressive clinicopathologic characteristics in human cancer. Cancer Res. 2012;72(14). doi: 10.1158/0008-5472.

21. Chen L, Li M, Li Q, Wang CJ, Xie SQ. DKK1 promotes hepatocellular carcinoma cell migration and invasion through $\beta$-catenin/MMP7 signaling pathway. Mol Cancer. 2013;12:157.

22. Popescu NC, Goodison S. Deleted in liver cancer-1 (DLC1): an emerging metastasis suppressor gene. Mol Diagn Ther. 2014;18(3):293-302.

23. Ko FC, Ping Yam JW. Regulation of deleted in liver cancer 1 tumor suppressor by protein-protein interactions and phosphorylation. Int J Cancer. 2014;135(2):264-9.

24. Feng X, Li C, Liu W, Chen H, Zhou W, Wang L, et al. DLC-1, a candidate tumor suppressor gene, inhibits the proliferation, migration and tumorigenicity of human nasopharyngeal carcinoma cells. Int J Oncol. 2013;42(6):1973-84.

25. Liao YC, Lo SH. Deleted in liver cancer-1 (DLC-1): a tumor suppressor not just for liver. Int J Biochem Cell Biol. 2008;40(5):843-7.

26. Goggs R, Williams CM, Mellor H, Poole AW. Platelet Rho GTPases-a focus on novel players, roles and relationships. Biochem J. 2015;466(3):431-442. 
27. Sheikh KA. Autoantobodies activate small GTPase RhoA to modulate neurite outgrowth. Small GTPases. 2011;2(4):233-238.

28. Wang Y, Lei R, Zhuang X, Zhang N, Pan H, Li G, et al. DLC1-dependent parathyroid hormone-like hormone inhibition suppresses breast cancer bone metastasis. J Clin Invest. 2014;124(4):1646-59.

29. Liao W, Huang W, Guo Y, Xin M, Fu X. Testosterone promotes vascular endothelial cell migration via upregulation of ROCK-2/moesincascade. Mol Biol Rep. 2013;40(12):6729-635.

30. Wang J, Liu H, Chen B, Li Q, Huang X, Wang L, et al. RhoA/ROCK-dependent moesin phosphorylation regulates AGE-induced endothelial cellular response. Cardiovasc Diabetol. 2012;11:7.

Submit your next manuscript to BioMed Central and we will help you at every step:

- We accept pre-submission inquiries

- Our selector tool helps you to find the most relevant journal

- We provide round the clock customer support

- Convenient online submission

- Thorough peer review

- Inclusion in PubMed and all major indexing services

- Maximum visibility for your research

Submit your manuscript at www.biomedcentral.com/submit
) Biomed Central 